\title{
Final Stage of the (Charge-Transfer Process in Charge-Coupled Devices
}

\author{
YOSHIAKI DAIMON, AMR M. MOHSEN, AND T. C. MCGILL, MEMber, IEeE
}

\begin{abstract}
The final stages of transfer of charge from under a storage gate is formulated analytically including both fringing-field induced drift and diffusion. Analytic solutions to these equations are presented for constant fringing fields, and a system of equations for spatially varying fields is developed. Approximate solutions for spatially varying fringing fields, when combined with a lumpedparameter model of the self-induced field effects, are shown to give a reasonably accurate representation of the free-charge transfer process.
\end{abstract}

\section{INTRODUCTION}

$\mathbf{E}$ ARLY descriptions [1], [2] of the charge transfer in charge-coupled devices (CCD) assumed that duringr the final stage of the charge transfer, the mechanism of transfer would be diffusion from under the storage gate. However, subsequent studies of the surface-potential profiles under the gates [3] indicated that fringing-field induced drift could act as an additional charge-transfe:: mechanism. In certain designs of CCD, this second mechanism can actually act as the dominant mechanism fo: transfer and enhance the rate of transfer during the final stage of charge transfer [4], [5].

These 2 mechanisms of transfer are characterized by time constants, the thermal-diffusion time constant, $\tau_{\text {th }}$ and the single-carrier transit time constant, $\tau_{\text {tr }}$. In the case when the fringing fields are 0 , the final stages of the diffusion processes are characterized by a profile which is a cosine function in shape and which decays exponentially with a time constant given by

$$
\tau_{\text {th }}=\frac{4 L^{2}}{\pi^{2} D}
$$

where $L$ is the length of the storage electrode, and $D$ is the diffusion constant.

On the other hand, if we neglect diffusion phenomenon, the charge remaining under the storage gate will be swept out in a single-carrier transit time

$$
\tau_{\mathrm{tr}}=\int_{0}^{L} \frac{d x}{\mu E(x)} .
$$

In this paper, we study these results analytically. We show that it is possible to obtain an analytic solution of

Manuscript received May 29, 1973. This work was supported in part by the Office of Naval Research under Grant N00014-67-A0094-0032 and the Naval Research Laboratories under Grant 00173-3-006252.

Y. Daimon and T. C. McGill are with the California Institute of Technology, Pasadena, Calif. 91109.

A. M. Mohsen is with Bell Laboratories, Murray Hill, N. J. 07060. the continuity equation in which the combined effects of diffusion and a uniform fringing field are included. A set of equations for spatially varying fringing fields are developed to show that the analytic solution for spatially varying fields can also be written in a form analogous to that for constant fringing fields.

The standard yariational method is applied to obtain an approximate analytic expression for the characteristic time constants for spatially varying fringing fields. Selfinduced drift terms are included by using an approximate lumped-circuit model.

\section{TRANSPORT DYNAMICS}

The transport dynamics along the insulator-semiconductor interface are described by the continuity equation

$$
\frac{\partial q}{\partial t}+\frac{\partial}{\partial x} J_{x}=0
$$

and the diffusion equation

$$
J_{x}=-D \frac{\partial q}{\partial x}+\mu q\left(-\frac{\partial \phi_{s}}{\partial x}\right)
$$

where $q$ is the surface-charge density, $\phi_{s}$ is the surface potential, and $x$ is the distance along the interface in the direction of charge transfer. $J_{x}$ is the sheet-current density.

In this paper, we want to consider the solution to (3) for boundary conditions and approximations appropriate to the case where the storage gate contains a small amount of charge. This condition will arise in the final stage of the charge transfer, in the complete charge-transfer mode [5], or when the CCD is operated in a low-level injection application, such as low light-level imaging. In these cases, we can to the first approximation neglect the selfinduced field terms.

When a CCD is operated in the complete chargetransfer mode, detailed numerical simulation of the transport dynamics under all the relevant gate electrodes and interelectrodes regions show that the charge transfer in the last stages of the transfer process can be approximately described by the discharge of the storage gate with an almost perfect sink at one end [5]. Therefore, we have considered the solution of (3) for the discharge of the storage gate using the boundary conditions

$$
\begin{aligned}
q(t, L) & =0, \quad \text { for } t>0 \\
J_{x}(t, 0) & =0, \quad \text { for } t>0 .
\end{aligned}
$$

Condition (4a) corresponds to assuming a perfect sink at 
the right-hand end of the storage gate $x=L ;(4 \mathrm{~b})$ corresponds to assuming that no current flows out of the bucket under the storage gate through the edge at $x=0$.

\section{CONSTANT FRINGING FIELD}

Assuming a constant fringing field $E$ under the storage gate and neglecting the self-induced field term, the residual surface-charge profile under the storage gate is given by solving (3) to obtain

$$
\begin{aligned}
q(t, x)=\sum_{n=1}^{\infty} A_{n} \exp & \left(\frac{E x}{2 k T}\right) \\
& \cdot \sin \left[\frac{\pi}{2} C_{n}\left(1-\frac{x}{L}\right)\right] \exp \left(-\frac{t}{\tau_{n}}\right)
\end{aligned}
$$

where $k T$ is the thermal voltage and $n$ is a summing index. The solution is given by a Fourier expansion multiplied by a common function, $\exp (E x / 2 k T)$, with constants $C_{n}, \tau_{n}$, and $\boldsymbol{A}_{n}$ to be determined as follows.

$C_{n}$ is determined by the boundary condition at $x=0$ and is given by solving the transcendental equation (see Fig. 1)

$$
\tan \left(\frac{\pi}{2} C_{n}\right)+\left(\frac{k T}{L E}\right) \pi C_{n}=0
$$

where $C_{n}$ is in the range given by

$$
2 n-1 \leq C_{n}<2 n, \quad \text { for } n=1,2, \cdots .
$$

$\tau_{n}$ is given by substituting (5) into (3) to obtain

$$
\frac{1}{\tau_{n}}=C_{n}^{2} \frac{\pi^{2} D}{4 L^{2}}+\frac{(\mu E)^{2}}{4 D} .
$$

Noting that the exponential term, $\exp (E x / 2 k T)$ in (5) can be taken outside of the summation, we then obtain

$$
\begin{array}{r}
A_{n}=\frac{2}{L\left[1-\left(\sin \pi C_{n}\right) / \pi C_{n}\right]} \int_{0}^{L} q(0, x) \exp \left(\frac{-E x}{2 k T}\right) \\
\cdot \sin \left[\frac{\pi}{2} C_{n}\left(1-\frac{x}{L}\right)\right] d x
\end{array}
$$

where use of the boundary condition (6a) has been made.

Detailed numerical simulations [4], [5] of charge transfer, including the effects of fringing fields, show that the profile of charge changes for a single-carrier transit time and then becomes stationary with an exponential time decay of the amplitude. This result is easily understood in light of the solution presented in the previous discussion. From (7) and (6b), one can see that $\tau_{n}$ is a decreasing function of $n$. Hence, for reasonably smooth initial charge distributions which produce finite values of $A_{n}$ which either remain relatively constant or decrease with increasing $n$, we expect that eventually the first term in the series in (5) dominates the series, and $q(t, x)$ can be approximated by

$$
q(t, x) \approx A_{1} \exp \left(\frac{E x}{2 k T}\right) \sin \left[\frac{\pi}{2} C_{1}\left(1-\frac{x}{L}\right)\right] \exp \left(-\frac{t}{\tau_{f}}\right)
$$

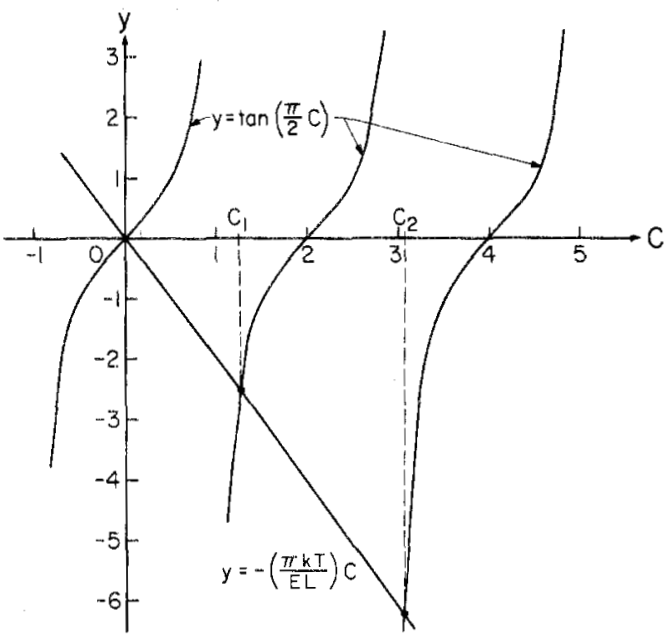

Fig. 1. The graph illustrates how to obtain $C_{1}$ and $C_{2}$ defined by (6a). Note $1<C_{1}<2$ and $3<C_{2}<4$ for any value of $L E / k T$. This is the condition given by $(6 \mathrm{~b})$ for numbering the root $C_{n}$ (the intersection) of the straight line with each branch of the tangent function.

where we have replaced $\tau_{1}$ by $\tau_{f}$ to indicate that it is the time constant characterizing the final decay of the charge. Using (7), we find that

$$
\frac{1}{\tau_{f}}=C_{1}^{2} \frac{\pi^{2} D}{4 L^{2}}+\frac{(\mu E)^{2}}{4 D} .
$$

Hence, we find for times which are greater than some as yet to be determined time (see Section IV), the charge profile remains constant, and the amplitude decays exponentially with time. The charge profiles at several different times are shown in Figs. 2 and 3 to illustrate the details of the charge transfer.

Determination of the value of $\tau_{j}$ depends upon the value of $C_{1}$. The results of a numerical solution of (6a) for $C_{1}$, as a function of the dimensionless parameter $E L / k T$, are plotted in Fig. 4. From this plot, we see that $C_{1}$ ranges from 1 for $E L / k T=0$ to a value of 2 as $E L / k T$ approaches infinity. (Note the first term in (6a) is negative.)

To compare this final decay constant with the 2 characteristic times defined in Section I, we have computed the value of the ratio of $\tau_{f}$ to $\tau_{\text {th }}$ to be

$$
\frac{\tau_{f}}{\tau_{\text {th }}}=\frac{1}{C_{1}^{2}+(L E / \pi k T)^{2}}
$$

and the value of the ratio of $\tau_{f}$ to $\tau_{\mathrm{tr}}$ to be

$$
\frac{\tau_{f}}{\tau_{\text {tr }}}=\frac{4 L E / \pi^{2} k T}{C_{1}^{2}+(L E / \pi k T)^{2}} .
$$

These ratios as a function of $L E / k T$ are plotted in Fig. 5 .

\section{STATIONARY PROFILE WITH CONSTANT FRINGING FIELD}

According to the detailed numerical simulation [4], [5] of charge transfer under the influence of fringing fields, the charge profile under the storage gate drifts for a 

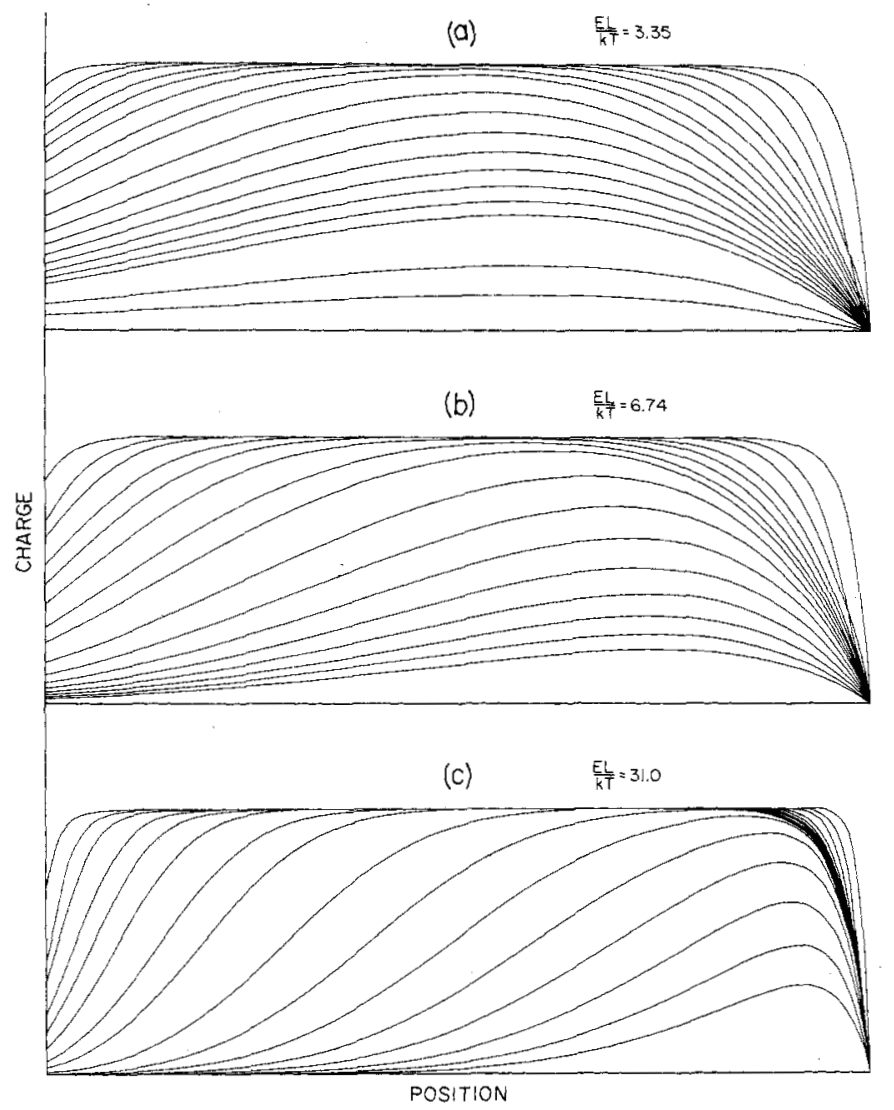

Fig. 2. The details of the charge decay at different instances a:e illustrated for three different values $E l / k T$ of constant fringin field strength. The initial charge profile is taken to be uniforr. The total initial charge is 70 percent of the full bucket charge $\left(14.6 \mathrm{C} / \mu^{2}\right)$. Note in all three cases, the relative charge profile becomes stationary within a single-carrier transit time. The position of the peak of the final charge profile is given exactly bosition of the peak of the (a) $E l / k T=3.35$. The total number of profiles shown is 18. The corresponding times are $0.01,0.02,0.03,0.05,0.07,0.10$, $0.15,0.2,0.3,0.4,0.5,0.6,0.7,0.8,0.9,1.0,1.5$, and 2.0 of a singlecarrier transit time. (b) $E l / k T=6.74$. The total number of profiles shown is 16. The corresponding times are similar to Fig. 2(a). The profiles at $t=1.5$ and 2.0 are deleted. (c) $E l / k T=$ 31.0. The total number of profiles shown is 16 . The correspondiag times are the same as Fig. $2(\mathrm{~b})$.

single-carrier transit time and then becomes stationary. No matter how strong the fringing fields are for reasonable initial charge distributions, such as uniform or a cosine shape, in the final stage of charge transfer, the stationary profile results eventually because of the thermal-diffusion mechanism. This is to say that the first term in the infinite series in (5) becomes the dominant one within an elapsad time, $t$, of the order of a single-carrier transit time.

We now return to the question of what is the value of the lower time limit for the validity of approximation of keeping only the first term in the series in (5). To gauge this time, we consider the ratio $r_{12}$ of the first two terms in the series in (5). Assuming that $A_{1}$ is the same as $A_{2}$ and neglecting spatial variation, this ratio is given by

$$
r_{12}=\exp \left[\frac{t}{\tau_{\text {th }}}\left(C_{2}^{2}-C_{1}^{2}\right)\right]
$$

where use of (7) has been made. In terms of the single- carrier transit time, this ratio can be written as

$$
r_{12}=\exp \left[\frac{t}{\tau_{\text {tr }}} \frac{\pi^{2} k T}{4 L E}\left(C_{2}^{2}-C_{1}^{2}\right)\right]
$$

Taking as our criterion the fact that the value of the exponent in this expression is greater than one, we find that (9) is valid for

$$
t>\tau_{\mathrm{tr}}\left[\frac{4 E L / \pi^{2} k T}{C_{2}^{2}-C_{1}^{2}}\right] .
$$

The right-hand side of this inequality is a multiple of the $\tau_{\text {th }}$, with the constant multiplying the transit time depending on the value of the parameter $E L / k T$. The value of this multiplier can be approximated as follows.

The value of $C_{2}^{2}-C_{1}^{2}$ varies monotonically between 8 when $E L / k T$ is 0 to 12 when $E L / k T$ approaches infinity. Thus we can replace the inequality by

$$
t>\tau_{\operatorname{tr}}\left(\frac{E L}{k T}\right) / 2 \pi^{2}
$$

This inequality shows that for $E L / k T$ between 0 and about 30 (the values normally encountered in devices), the approximation is valid for times greater than a singlecarrier transit time. Hence, the results of the numerical simulations are in good agreement with the analytical results obtained here, and we can describe the final charge profile by (9).

The peak position of the charge packet, $x_{\text {peak }}$, after it becomes stationary under the storage gate, is given by differentiating (9) with respect to $x$ and setting the resulting equation to be 0 . Then using the condition (6a), we obtain

$$
x_{\text {peak }}=2 L\left(1-\frac{1}{C_{1}}\right) .
$$

This expression shows the peak-position varies with the strength of the fringing field. For 0 field, $C_{1}$ is unity. The surface-charge profile is a cosine function with a maximum at $x=0$, and it decays exponentially with the time constant equal to the thermal-diffusion time constant.

For large fringing fields, the value of $C_{1}$ approaches 2 as shown in Fig. 4, but never becomes larger than 2. For extremely large fringing fields, $C_{1} \approx 2$ and $x_{\text {peak }} \approx L$, implying that the peak position approaches the sink edge at $x=L$. At times, the exponential decay is observed satisfying the inequality in (12), and the characteristic decay time is a factor approximately $\frac{1}{3}$ of the single-carrier transit time, as we observe in Fig. 5. Figs. 2 and 3 illustrate the details of the charge decay as we have discussed so far.

\section{SPATIALLY VARYING FRINGING FIELDS}

We now note that the constant and spatially varying fringing fields both give similar charge-decay characteristics. This is to say that the analytic solutions of both spatially varying and constant fringing fields can be written in similar forms. Both solutions can be expressed by infinite series, and as time elapses, the term with the 


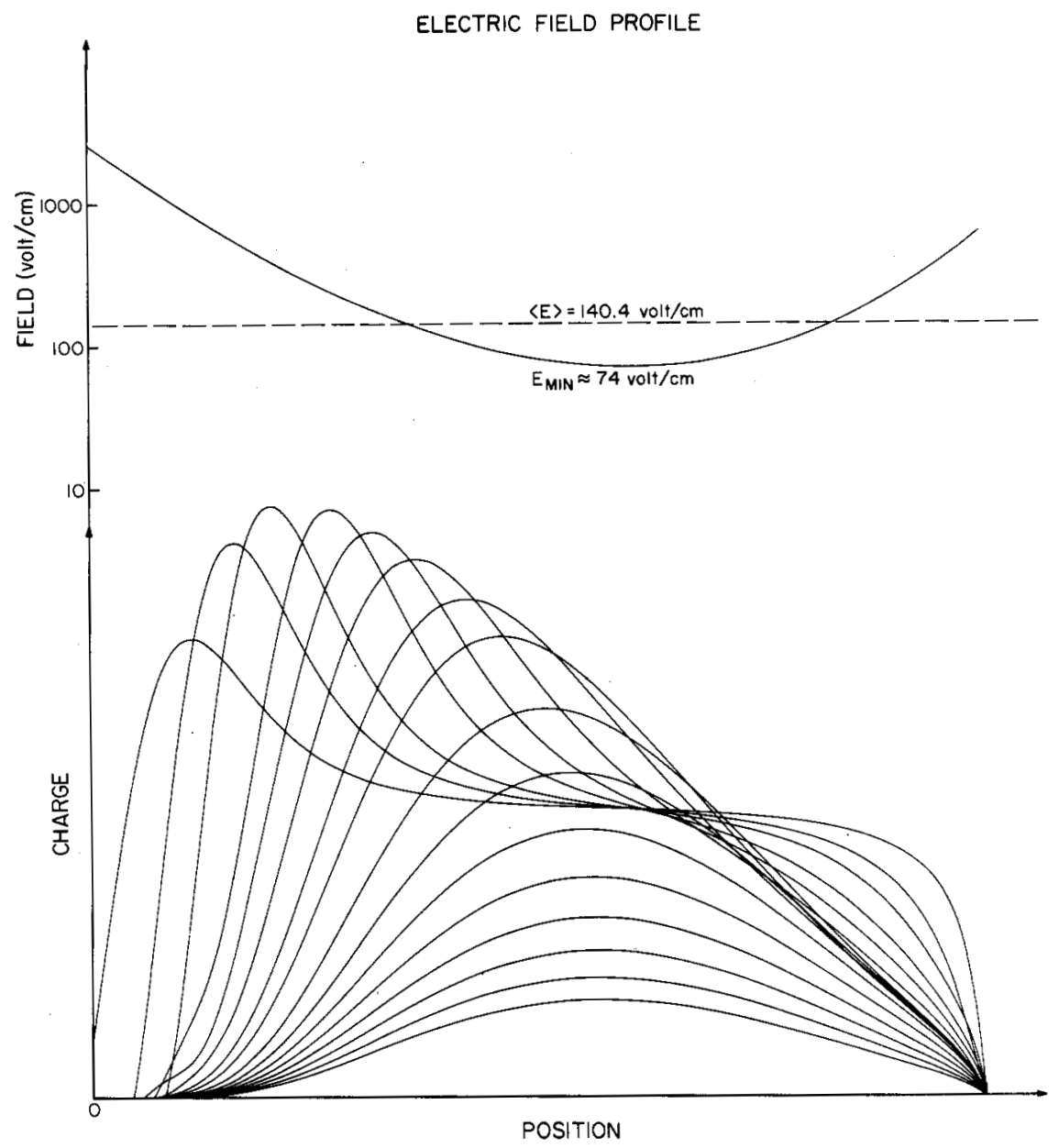

Fig. 3. The details of the charge decay at different stages. The condition is similar to the results of Fig. 2 except the fringing field is spatially varying in this case. An average fringing field computed by (2) is $140 \mathrm{~V} / \mathrm{cm}$. The minimum field is $74 \mathrm{~V} / \mathrm{cm}$. The total number of profiles shown is 16 . The corresponding times are the same as Fig. 2(b) and (c).

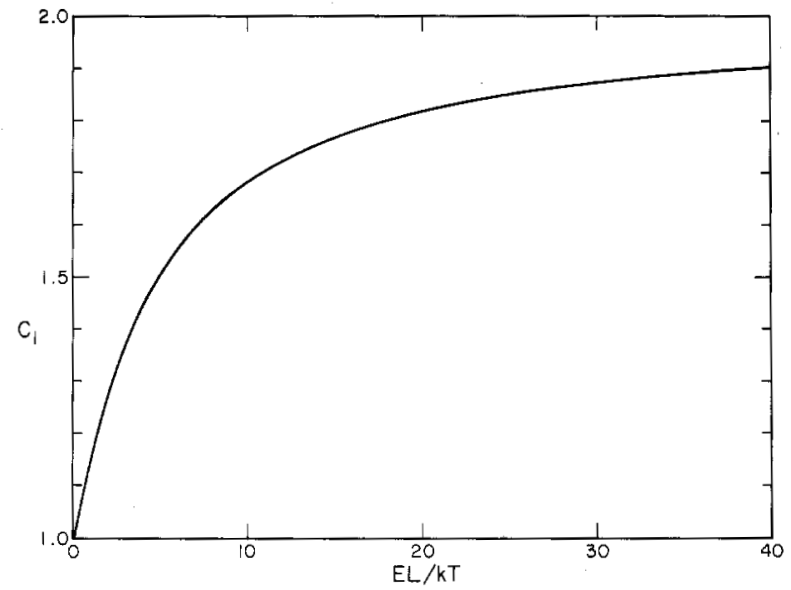

Fig. 4. Values of $C_{1}$ defined by (6), plotted against the normalized fringing-field strength $E L / k T$.

largest time constant $\tau_{1}$ becomes dominant, resulting in the exponential decay characteristics and the constant charge profile. In this case, it is convenient to work with

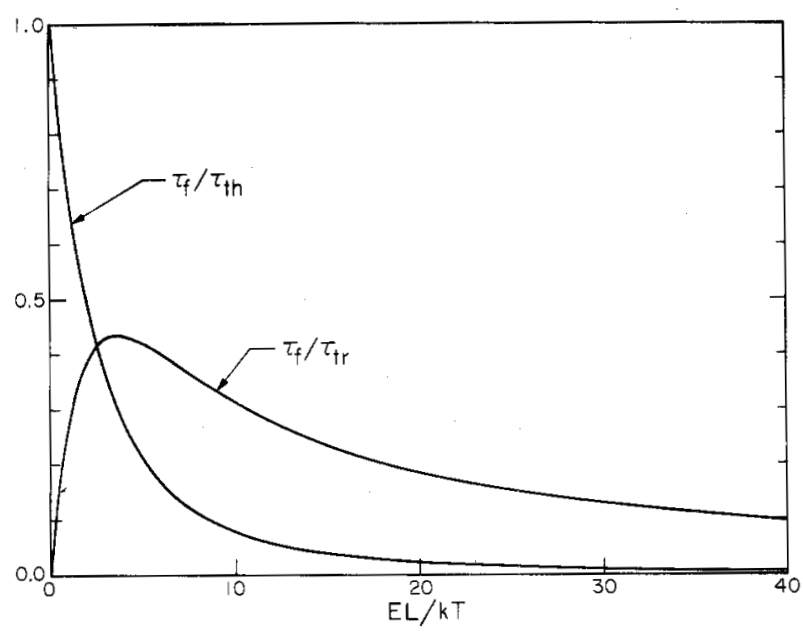

Fig. 5. The final decay time constant, $\tau_{f}$, normalized by the singlecarrier transit time $\tau_{\mathrm{tr}}$ and by the thermal-diffusion time constant $\tau_{\text {th }}$, plotted against the normalized fringing-field strength $E L / k T$.

$Q(t, x)$, as seen by

$$
Q(t, x)=\int_{0}^{x} q\left(t, x^{\prime}\right) d x^{\prime} .
$$


Using the definition for $Q$ and the fact that current flows only out of one end of the gate (see (4b)), we can write (3) as

$$
\frac{\partial Q}{\partial t}=D \frac{\partial^{2} Q}{\partial x^{2}}-\mu E(x) \frac{\partial Q}{\partial x}
$$

where again, we have replaced $\tau_{1}$ by $\tau_{f}$.

The largest time constant $\tau_{f}$ could, in general, be obtained by solving (19) and (20). However, a good estimate can be obtained from the standard variational procedure for lowest eigenvalues [8]. According to this procedure, the exact value of $\tau_{f}$ is obtained by minimizing

$$
\frac{1}{\tau_{1}}=\left[D \int_{0}^{L}\left[d \gamma_{1} / d x\right]^{2} d x+(\mu / 2) \int_{0}^{L}\left[E^{\prime 2}(x) / 2 k T-d E / d x\right] \gamma_{1}^{2}(x) d x\right] / \int_{0}^{L} \gamma_{1}^{2}(x) d x
$$

where the boundary condition equivalent to (4a) is given by

$$
\left.\frac{\partial Q(t, x)}{\partial x}\right|_{x=L}=0
$$

The boundary condition ( $4 b)$ is used to derive $(16)$. We now have instead, a different boundary condition at $x=0$, which follows directly by the definition of $Q(t, x)$ given by $(1 \tilde{j})$ and is seen as

$$
Q(t, 0)=0, \quad \text { for all } t
$$

To eliminate the first derivative from (16), we introduce the following transformation:

$$
Q(t, x)=\exp \left(\frac{1}{2 k T} \int_{0}^{x} E(x) d x\right) R(t, x) .
$$

Then, (16) becomes

$$
\frac{\partial R}{\partial t}=D \frac{\partial^{2} R}{\partial x^{2}}-\frac{\mu}{2}\left[\frac{E^{2}(x)}{2 k T}-\frac{d E}{d x}\right] R
$$

with the new boundary conditions given by

$$
\frac{\partial R}{\partial x}+\frac{E(x)}{2 k T} R=0, \quad \text { at } X=L
$$

and

$$
R(t, 0)=0, \quad \text { for all } t .
$$

The solution of (19) together with the boundary conditions is given by an infinite series of the form

$$
R(t, x)=\sum_{n=1}^{\infty} B_{n} \gamma_{n}(x) \exp \left(-t / \tau_{n}\right)
$$

where $B_{n}$ is to be determined by the initial values of $R(0, x), \gamma_{n}(x)$ and $\tau_{n}$ are the eigenfunctions and eigenvalues, respectively, of the eigenvalue problem given in (19) and (20).

The results in the case of constant fringing fields combined with the results of detailed numerical simulations suggest strongly that for times which are a few times the single-carrier transit time, we can approximate the scries in (21) by the first term and write

$Q(t, x)=B_{1} \exp \left(\frac{1}{2 k T} \int_{0}^{x} E(x) d x\right) \gamma_{1}(x) \exp \left(-t / \tau_{f}\right)$
The solution of (19) with $E(x)$ constant suggests the trial function for the first eigenfunction.

$$
\gamma_{1}(x)=\sin \left[\frac{\pi C_{1} x}{2 L}\right] .
$$

This trial function must satisfy the boundary conditions of $(20)$. That is, the value of $C_{1}$ is to be determined by the strength of the fringing field $E(L)$ at the sink edge of the gate (see (20a)). Since the fringing fields at the ends of storage gates are very large [3], [5], we have

$$
E(L) \gg k T / L \text {. }
$$

Hence, substituting $\gamma_{1}(x)$ of (24) into $R$ of (20a), we obtain

$$
-\tan \left(\frac{\pi C_{1}}{2}\right)=\pi C_{1} \frac{k T}{L E(L)} \ll 1
$$

and hence,

$$
C_{1} \approx 2
$$

With this value of $C_{1}$, substituting the trial function given by (24) into (23), we obtain

$$
\frac{1}{\tau_{1}}=\frac{4}{\tau_{\text {th }}}+\frac{\mu^{2} E_{\mathrm{eq}}^{2}}{4 D}
$$

$E_{\mathrm{eq}}$ is an equivalent constant fringing field for the spatially varying fringing field $E(x)$ and is given by

$$
E_{\mathrm{eq}}{ }^{2}=\frac{2}{L} \int_{0}^{L}\left[E^{2}(x)-2 k T \frac{d E}{d x}\right] \sin ^{2}\left[\frac{\pi x}{L}\right] d x .
$$

Note that, due to the weighting function $\sin ^{2}(\pi x / L)$, the integral vanishes at both ends of the gate; $x=0$ and $L$. The contribution of the intergrand at the ends of the storage gate is relatively small, but the fringing fields at the positions of high-charge concentration are weighted heavily in the integral. If the fringing field is slowly varying and is at its minimum value $E_{\mathrm{m} \text { in }}$ under most of the storage gate (except at the ends), then we obtain $E(x) \approx$ $E_{\min }$ and $d E / d X \approx 0$. Hence, from $(27 \mathrm{~b})$, we have $E_{\text {eq }} \approx E_{\mathrm{min}}$. Finally, we obtain an approximate analytic formula of the time constant for spatially varying fringing fields, which is

$$
\frac{1}{\tau_{f}}=\frac{1}{\tau_{1}} \approx \frac{4}{\tau_{\text {th }}}+\frac{\mu^{2} E_{\min ^{2}}^{2}}{4 D} .
$$

Care must be taken in applying this formula. This formula 
was obtained for slowly varying fringing fields under most of the storage gate, and other configurations may give different fringing-field profiles leading to different results.

For example, in CCD structures with short storage-gate length $L, E_{\mathrm{eq}}$ may be a few times larger than $E_{\mathrm{min}}$. In this case, (27b) could be used to obtain $E_{\text {eq }}$ if the fringingfield profile is known. For short storage-gate length $L$, the spatial dependence of the fringing fields can be approximated by $[3]$

$$
\begin{aligned}
& E(x)=\frac{E_{\mathrm{min}} L}{2 X}, \quad \text { for } \quad 0 \leq X \leq L / 2 \\
& =\frac{E_{\mathrm{min}} L}{2(L-X)}, \quad \text { for } \quad L / 2 \leq X \leq L
\end{aligned}
$$

Substituting (29) into (27b), we obtain $E_{\mathrm{eq}}=1.95 E_{\mathrm{min}}$ for the same trial function $r_{1}(x)$ given by $(24)$. In either case, as seen in (28), we note that the reduction of the final decay time constant by the fringing-field strength is quadratic rather than linear.

If we include the nonlinear self-induced field drift, exact analytic solutions of (3) become difficult. However, using a lumped-circuit model, ${ }^{1}$ the charge-transfer characteristics can be obtained by solving the discharge equation $[5],[6]$

$$
J_{x}=-\frac{d Q(t, L)}{d t}
$$

where $J_{x}$ is the steady-state discharge current density assumed constant across the gate, and $Q(t, L)$ is the total charge under the gate.

The relation between the surface potential $\phi_{s}$ and surface-charge density $q$, under the transfer gate, is given according to the gradual channel approximation [4], [5]

$$
\phi_{s}=\phi_{s_{0}}+\frac{q}{C}
$$

where $\phi_{\varepsilon_{0}}$ is the surface potential with no charge, and $C$ is the effective oxide- and depletion-layer capacitance per unit area. When the fringing fields are negligible compared to the self-induced field, we have

$$
\frac{\partial \phi_{s}}{\partial x} \approx \frac{1}{C} \frac{\partial q}{\partial x}
$$

Then, if the difference in the surface potential between the beginning and end of the gate is $V$, by integrating the diffusion equation (3b) over space, we obtain [5], [7]

$$
J_{x}=\frac{\mu C}{2 L}\left[V^{2}+2 k T V\right]
$$

where, we note

$$
q(0)=C V
$$

Since $J_{x}$ in $(3 \mathrm{~b})$ is assumed constant for $0 \leq x \leq L$, we

\footnotetext{
${ }^{1}$ According to this model, the storage gate is considered, in this case, as a capacitor discharged through a transfer channel which is the same storage gate.
}

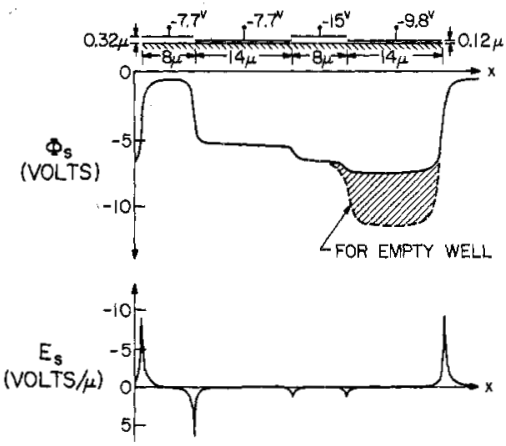

Fig. 6. The surface potential and electric field along the Si-SiO interface obtained from the solution of the two-dimensional Poisson's equation. The electrode voltages correspond to the latter stages of the charge transfer, ${ }^{2}$ with a signal charge in the receiving storage gate. The substrate doping is $10^{14}$ donors $/ \mathrm{cm}^{3}$.

The fixed surface-state charge is $3.6 \times 10^{11} / \mathrm{cm}^{2}$, in this calculation.

obtain $q(x)$ for $0 \leq x \leq L$. Then, by integrating $q(x)$, we obtain

$$
Q(t, L)={ }_{3}^{2} L C V \frac{V+3 k T / 2}{V+2 k T} .
$$

Then, the solution of $(30 \mathrm{a})$ is of the form

$\frac{Q(t, L)}{Q(0, L)}$

$$
=\frac{\exp \left(-t / \tau_{f}\right)}{1+Q(0, L) / \frac{2}{3} C L(1 / 2 k T)\left(\tau_{f} / \tau_{\text {th }}\right)\left[1-\exp \left(-t / \tau_{f}\right)\right]}
$$

where $Q(0, L)$ is the initial total charge under the storage gate, and $\tau_{f}$ is given by $(28)$.

\section{NUMERICAL RESULTS}

The exact fringing-field profile can be obtained by solving the two-dimensional Poisson equation for the CCD structure with the applied gate voltages. In Fig. 6, we have plotted the surface potential and surface-potential gradient along the semiconductor-insulator interface. The voltages on the gate electrodes (see Fig. 6) are those corresponding to the last stage of the charge transfer. Most of the signal charge was taken to be in the receiving storage electrode. ${ }^{2}$ Periodic boundary conditions were used. The minimum fringing field $E_{\mathrm{m} \text { in }}$, in this case, is equal to $74 \mathrm{~V} / \mathrm{cm}$.

To check the accuracy of the approximate solution for $\tau_{f}$ given by (28), we have solved (3) numerically for the fringing-field profile given in Fig. 6 [2]. The full-line curve in Fig. 7 represents the numerically calculated residual charge under the storage gate versus transfer time with the self-induced fields. The dashed-line curve in Fig. 7 is the residual charge calculated using (32). The value

${ }^{2}$ For two-phase push-clock scheme, the actual receiving gate voltage is $-15.0 \mathrm{~V}$ instead of $-9.8 \mathrm{~V}$ in Fig. 3 , and the difference of $5.2 \mathrm{~V}$ corresponds to the amount of the signal charge present under the receiving gate. 


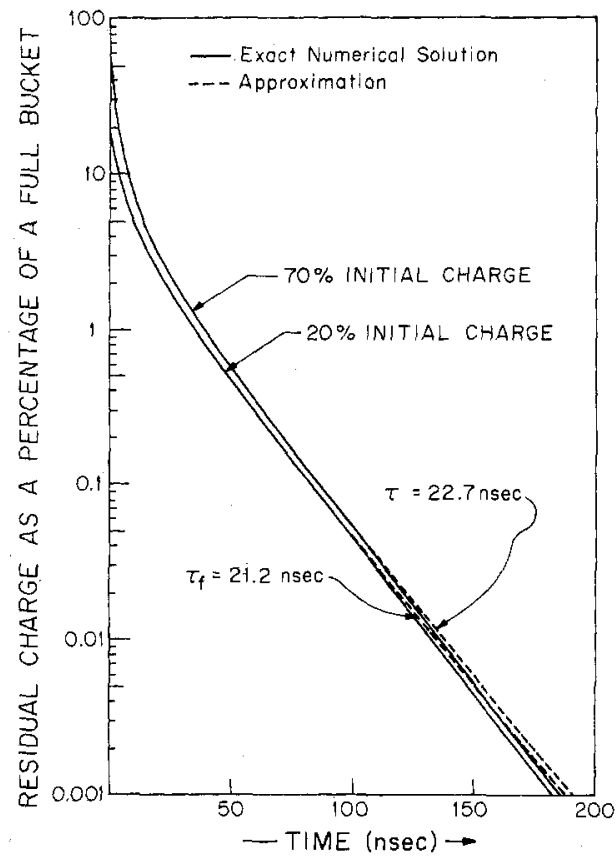

Fig. 7. Total residual charge under the left Si-source gate as a function of time. The effective oxide capacitance is $3.22 \mathrm{~F} / \mu^{2}$ and the full bucket charge is $14.6 \mathrm{C} / \mu^{2}$. The solid curve represents exact numerical solution of (3), and the dashed curve is an approximation by (32). $\tau_{f}$ is for the slope of the two parallel solid lines and $\tau$ for the dashed lines. The final slope (hence, also tixe final time constant) does not depend on the total amount of tie initial charge under the storage gate.

of the final decay time constant $\tau_{j}$ calculated from (23) is $22.7 \mathrm{~ns}$ compared to $21.2 \mathrm{~ns}$ obtained from the numerical solution.

\section{CONCLUSION}

Incomplete transfer of free charge in CCD with small amounts of charge to be transferred was characterizad analytically, including the effects of diffusion and fringing fields. We have found that, independent of the fringirgfield profile, the exponential decay characteristic is solely due to diffusion. However, the characteristic time constant $\tau_{f}$ for the decay depends on diffusion and fringing fields and is found to be always a fraction of the singlecarrier transit time $\tau_{\text {tr }}$ (see Fig. 5).

The standard variational procedure was applied to obtain an approximate analytic expression for the characteristic time constant (See (23)), and the expression was evaluated for spatially varying fringing fields (see (26)) which vary relatively slowly over most of the storage gate length but increase considerably at the edges of the gates. Such fringing-field profiles are typical for most miximum geometry CCD structures (minimum gate dimension of about $10 \mu$ ) and substrate doping greater than $10^{14} / \mathrm{cm}^{3}$. The constant and spatially varying fringing fields both were found to give exponential charge-decay characteristics. When the magnitude of the field becomes greater than a few times $k T / L$, the discharge is considerably enhanced by the fringing-field drift.

If the self-induced field terms are introduced, then, using a lumped-circuit model, the free-charge transfer process is given quite accurately by the formula given.

\section{ACKNOWLEDGMENT}

The authors wish to thank C. A. Mead and the reviewers whose valuable suggestions have greatly improved the readibility of this paper.

\section{REFERENCES}

[1] C. K. Kim and M. Lenzlinger, "Charge transfer in chargecoupled devices," J. Appl. Phys., vol. 42, pp. 3586-3594, 1971.

[2] R. J. Strain and N. L. Schryer, "A nonlinear diffusion analysis of charge-coupled device transfer," Bell Syst. Tech. J., vol. 50, no. 6, pp. 1721-1740, 1971 .

[3] J. E. Carnes, W. F. Kosonocky, and E. G. Ramberg, "Driftaiding fringing fields in charge-coupled devices," IEEE J. Solid-State Circuits, vol. SC-6, pp. 322-326, Oct. 1971.

[4] - "Free charge transfer in charge-coupled devices," IEEE Trans. Electron Devices, vol. ED-19, pp. 798-808, June 1972.

[5] A. M. Mohsen, T. C. McGill, and C. A. Mead, "Charge transfer in overlapping gates charge-coupled devices," IEEE J. SolidState Circuits, vol. SC-8, pp. 191-207, Mar. 1973.

[6] C. N. Berglund and K. K. Thornber, "A fundamental comparison of incomplete charge transfer in charge-transfer devices," Bell Syst. Tech. J., vol. 52, no. 2, pp. 147-182, 1973.

[7] - "Incomplete transfer in charge-transfer devices," IEEE J. Solid-State Circuits, vol. SC-8, pp. 108-116, Feb. 1973.

[8] F. Mathews and R. L. Walker, Mathematical Methods of Physics. New York: W. A. Benjamin, Inc., pp. 315-318, 1965. 